\title{
Comparative Study between Lateral Band Mobilization and Spiral Retinacular Ligament Reconstruction in Swan Neck Deformity
}

\author{
AHMED ABDELMOATY MOHAMED ZEINA, M.Sc.*; REDA A. YOUNIS, M.D.**; \\ HOSSAM ELDIN A. ISMAIL, M.D.**; AHMED M. BAHAAELDIN, M.D.** and \\ MOHAMMED RADWAN EL-HADIDY, M.D.** \\ The Department of Plastic Surgery*, Student Hospital and Plastic Surgery Department**, Faculty of Medicine, \\ Mansoura University
}

\begin{abstract}
Background: Sawn neck deformities of the fingers are challenging and difficult for treatment. Many techniques had been used with different success rates depending on the cause of deformity and the technique used for correction. Techniques deal with either lateral band mobilization or oblique retinacular ligament reconstruction (ORL) popularly used with many refinements.
\end{abstract}

Objectives: To evaluate which surgical maneuver is better for correction of swan neck deformity and to compare results of lateral band mobilization and oblique retincular ligament reconstruction.

Patients and Method: 11 patients suffered from swan neck deformities. 24 fingers after rheumatoid arthritis, 5 post-burn and 3 post-traumatic. Divided into 2 groups, 20 fingers were corrected by lateral band mobilization (Group A). 12 fingers underwent ORL reconstruction (Group B). Assessment was done by measurement of extension lag on DIP by goniometer after 6 months compared with that done pre-operatively.

Results: DIP extension lag improved with lateral band mobilization especially non-rheumatoid patients. But with ORL reconstruction the results were not satisfactorty as regard as entension lag and PIP hyperentension.

Conclusion: Lateral band mobilization is a reliable method for correction of swan neeck deformity especially with nonrheumatoid patients (burn and trauma), but long-term results of lateral band translocation in rheumatoid patients are disappointing, as regard as ORL reconstruction with tendon graft, it did not show satisfactory results in this study.

Key Words: Swan neck deformit - Lateral band mobilization - Oblique retinacular ligament reconstruction.

\section{INTRODUCTION}

Lateral bands are continuations of interosseus and lumbrical tendons that run along sides of the digit at the proximal phalanx. Dorsal Subluxtion of the lateral bands such as in swan neck deformity is prevented by the transverse retinacular ligaments (TRL) that run from the flexor tendon sheath to the volar rim of lateral bands [1]. Anatomically, the ORL originates from the volar middle third of the proximal phalanx and the PIP flexor sheath, passes dorsally deep to the TRL and joins the lateral aspects of the terminal extensor tendon distally [2]. Functionally, this ligament acts as a dynamic tenodesis, extending the DIP joint as the PIP joint is extended. Although transection of this ligament alone does not cause an appreciable DIP joint extensor lag, the ORL is assumed to play a role in the coordination of the PIP and DIP joint motion and to act as a retaining ligament for the terminal tendons over the dorsum of the finger [3].

Swan neck deformity can be disabling deformities in the fingers resulted from a muscle imbalance caused by over activity of the extrinsic wrist flexors combined with intrinsic muscle spasticity in the hand [4]. Weakness of the proximal interphalangeal joint (PIP) stabilizing mechanisms and increased power of the long extensors and intrinsic muscles cause hyperextension of the PIP joint and dorsal dislocation of the lateral bands of the extensor tendons. The changing geometry of the extensor apparatus elongates the lateral bands and shortens the proximal extensor tendon, causing drooping of the distal interphalangeal joint (DIP) and further hyperextension at the PIP joint. The ORL shifts dorsal to the axis of the PIP joint and a progressively worsening deformity occurs [5].

Zancolli [6] originally described lateral band translocation, the goal of which is to stabilize the 
PIP joint by transfer of one of the lateral bands to the flexor tendon sheath volar to the PIP joint axis. ORL reconstruction was first suggested by Littler (1966) [7]. The classic reconstruction involves dividing the lateral band at the base of the proximal phalanx. The band is dissected to its insertion and redirected volar to cleland's ligament to be secured to $A_{2}$ pulley. The lateral band may be routed across the palmar surface of the PIP joint and secured to the bone of the radial side of the proximal phalanx, for a more secure repair. A modification of this technique uses a tendon graft instead of the lateral band, if the lateral band is of poor quality Thompson [7]. The concept of ORL reconstruction is a dynamic tenodesis that improves the stability of both the DIP and PIP joints by linking the volar flexor sheath to the lateral aspect of the terminal tendon, thereby providing a mechanism for automatic DIP joint extension upon active PIP extension [3]. The aim of this study was to compare results of lateral band mobilization and ORL reconstruction using standard points of comparison. These points are improvement active range of motion at DIP and improvement of overall hand function.

\section{PATIENTS AND METHODS}

\section{Patients:}

After getting the approval from the Ethical Committee of Faculty of Medicine, Mansoura University, this interventional study had been performed on 14 patients (40) fingers (10 index fingers, 14 middle fingers, 10 ring fingers and 6 little fingers. The cause of swan neck deformity was rheumatoid arthritis in 32 fingers, burn in 5 fingers and 3 post traumatic fingers) from May 2016 to April 2018. Their mean age was 40.5 years and their mean postoperative follow-up was 6 months. For each patient, age, sex, medical and surgical history was recorded. All patients were requested to have pre-operative, 6 months postoperative DIP goniometer measurement. Patients with stiff PIP or DIP joints were excluded.

\section{Inclusion criteria:}

Mobile joints (PIP and DIP). Cooperative patient to follow instructions and continue physiotherapy.

\section{Exclusion criteria:}

Patients with who unwilling to accept procedure risk or unfit for surgery. Non-compliant Patients with physiotherapy.

\section{Methods:}

The approach for both groups through linear incisions on dorsal or volar aspects of the fingers that doesn't cross the flexion creases to avoid contracture scars. General anesthesia was used and tourniquet $100 \mathrm{mmHg}$ above systolic pressure for all patients.

Group A (lateral band mobilization): Skin was incised and exposure of both central slip and lateral bands. Dissection of lateral bands till DIP joint to ensure free DIP movement then by prolene 3/0 round needle simple sutures were taken into one or both lateral bands to be rotated volarly to the flexor tendon sheath or $\mathrm{A}_{2}$ pulley volar to the PIP joint axis. Additional fixation sutures were placed, if necessary, to adjust the intra-operative tension. Exposure and mobilization of the other lateral band is indicated if PIP hyperextension not corrected by one band mobilization. In our study we used prolene 3/0 with round needle due to less tissue reaction and inflammatory response than absorbable suture, also cannot be dissolved and keep tensile strength. (Fig. 1).

Group B (ORL reconstruction by tendon graft): Extensor indicies proprius (EIP) tendon was used as a graft donor. EIP arises from the distal third of the dorsal part of the body of ulna. It joins the ulnar side of the tendon of the extensor digitorum which belongs to the index finger. Anatomically, the ORL originates from the volar middle third of the proximal phalanx and the PIP flexor sheath, passes dorsally deep to the transverse retinacular ligament, and joins the lateral aspects of the terminal lateral band tendon distally. Our technique to suture the graft into volar aspect of the finger volar plate or FDS or $\mathrm{A}_{2}$ pulley, free end is rerouted to the lateral band, Ensuring corrected position of PIP and DIP (like anatomical origin and insertion) (Fig. 3).

\section{Post operative follow-up:}

Dressing was done by vaseline gauze and sterile gauze then cotton pad direct under the cast. Pins are inserted to maintain PIP flexion 2-3 weeks. When the pins are removed, dorsal splints are used to prevent recurrence of PIP hyperextension and imbalance. A plaster cast splint was applied with the PIP joints in 10 to 30 degrees flexion, the MCP joints in 50 to 70 degrees flexion, and the wrist in a 0 to 30 degrees extension for a period of 6 weeks. Stitchs had been removed after 10 days; followup for 6-12 months. 

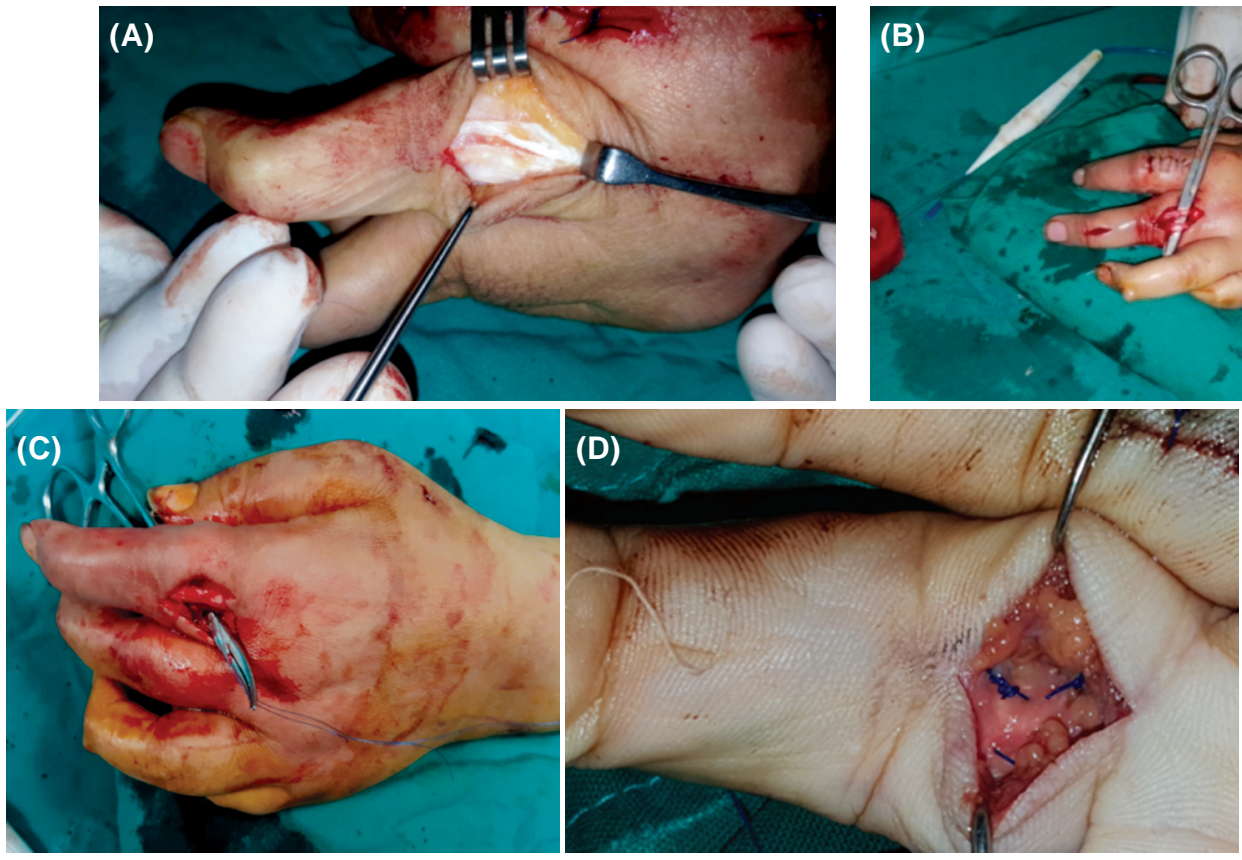

Fig. (1): Group A operative technique, (A) Exposure of lateral bands and central extensor tendon, (B) Both radial and ulnar bands (Note distal incision of middle finger for complete band freeings), (C) Simple stitch in ulnar band by prolene proximal to PIP joint and volar rotation of lateral band, (D) Volar fixation to $\mathrm{A}_{2}$ pulley.
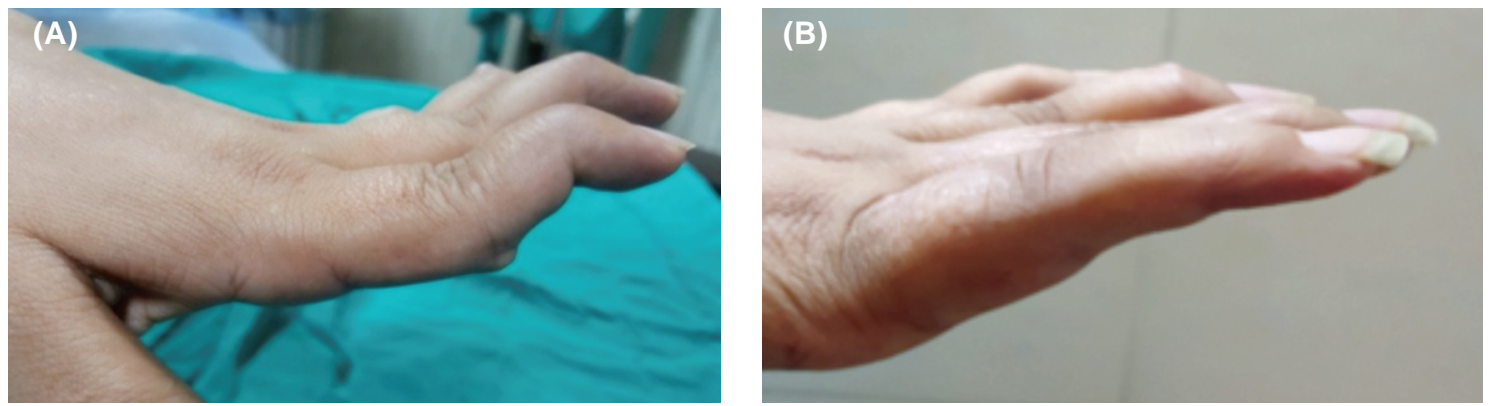

Fig. (2): Group (A) example: (A) Pre-operative lateral view, (B) Post-operative lateral view.
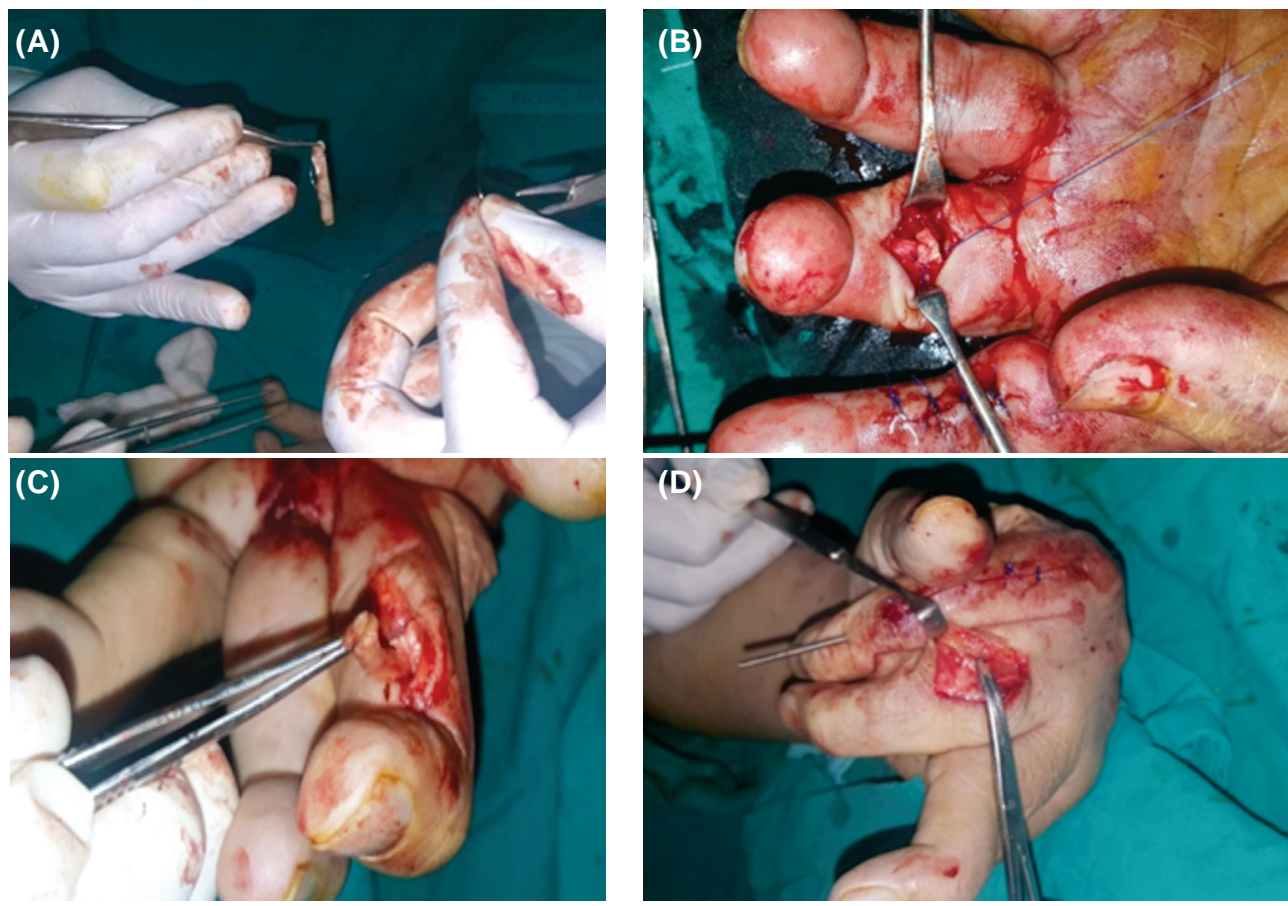

Fig. (3): Group B operative technique, (A) Suturing one end of the tendon graft, (B) Volar fixation of the tendon graft to flexor tendon sheath and $A_{2}$ pulley, (C) The free end of the graft, (D) Dorsal rotation of the graft ensuring correction of the PIP hyperextension. 

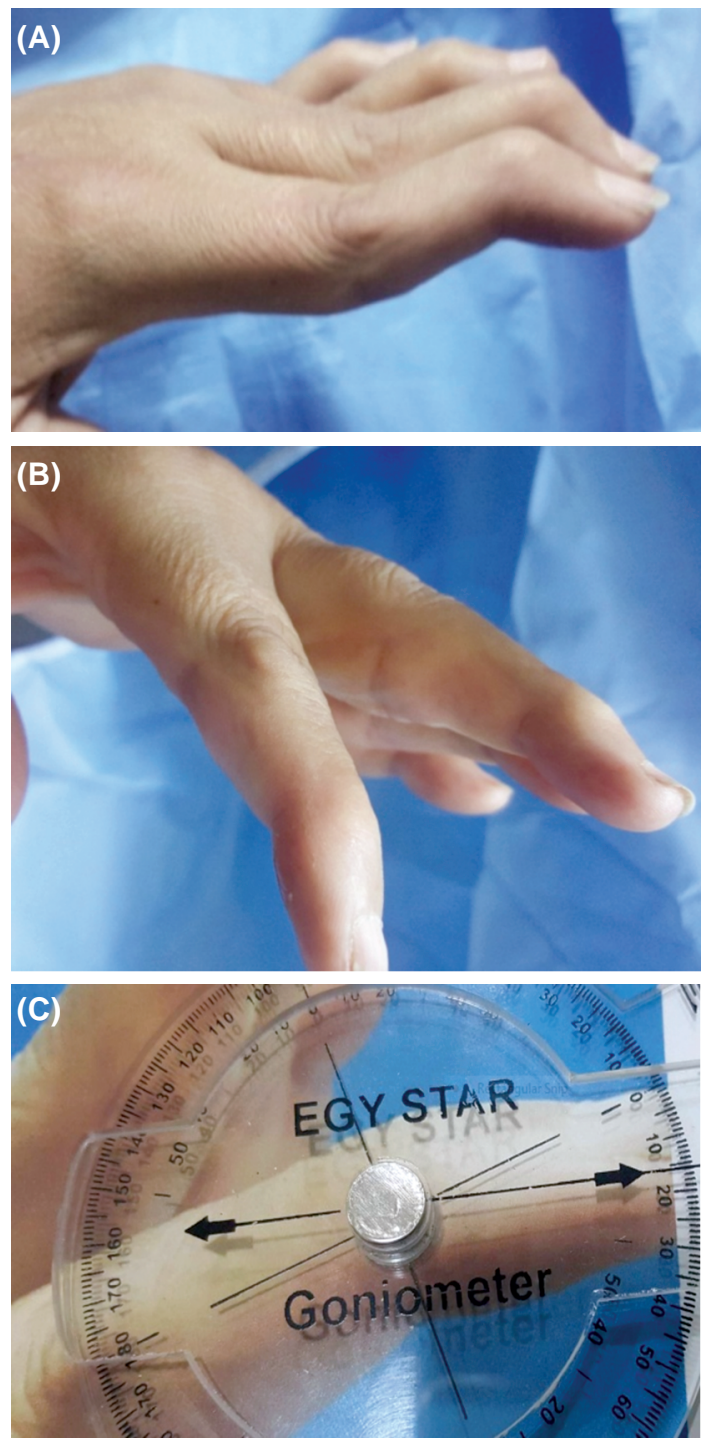

Fig. (4): Group (B) example: (A) Pre-operative lateral view, (B) Post-operative lateral view, (C) Post-opertive goniometer was $16^{\circ}$.

\section{RESULTS}

Among the 40 fingers (14 patients) whom had surgery, 8 fingers ( 3 patients) were lost to followup. This left 32 fingers for this study, our results based on 20 fingers were corrected by lateral band mobilization (group A) and 12 fingers reconstructed by tendon graft (group B). The results for goniometer assessment of DIP are listed in two sepatate tables also with the statistical significane of the results, Tables $(1,2)$ for group (A) while Tables $(3,4)$ for group $(B)$.

Group (A) results are shown in (Table 1).

p-value and statistical significance of group $(A)$ :

The statistical analysis of data done by using Excel program 2013 and IBM SPSS (Statistical
Package of Social Science) version 22, A p-value less than 0.05 was considered significant. The Unpaired $t$-test results shows: $p$-value equals $0.0019, t=3.3371$ and standard error of difference $=1.798$. By conventional criteria, this difference is considered to be statistically significant (for short term follow-up); statistical analysis is shown in Table (2).

\section{Group (B) results are shown in (Table 3).}

$p$-value and statistical significance of group $(B)$ :

$p$-value equals $0.0545, t=2.0311$ and standard error of difference $=5.088$ this difference is considered to be not statistically significant. Statistical analysis is shown in Table (4).

Table (1): Group (A) Goniometer measurements in degrees pre-operative and 6 months post-operative.

\begin{tabular}{cc}
\hline Pre-operative & Post-operative \\
\hline 24 & 6 \\
23 & 10 \\
22 & 12 \\
22 & 0 \\
14 & 11 \\
22 & 19 \\
22 & 18 \\
15 & 13 \\
16 & 3 \\
27 & 16 \\
28 & 24 \\
19 & 15 \\
17 & 15 \\
24 & 22 \\
29 & 24 \\
28 & 24 \\
28 & 24 \\
26 & 23 \\
25 & 22 \\
16 & 13 \\
\hline
\end{tabular}

Table (2): Statistical analysis of group A.

\begin{tabular}{lcc}
\hline Statistical data & $\begin{array}{c}\text { Pre- } \\
\text { operative }\end{array}$ & $\begin{array}{c}\text { Post- } \\
\text { operative }\end{array}$ \\
\hline Mean & 22.20 & 15.70 \\
SD (standard deviation) & 5.02 & 6.91 \\
SEM (standard deviation of mean) & 1.12 & 1.55 \\
Number & 20 & 20 \\
\hline
\end{tabular}

Table (3): Group (B) Goniometer measurements in degrees pre-operative and 6 months post-operative.

\begin{tabular}{cc}
\hline Pre-operative & Post-operative \\
\hline 59 & 16 \\
62 & 29 \\
62 & 62 \\
32 & 29 \\
30 & 29 \\
30 & 28 \\
32 & 30 \\
33 & 29 \\
33 & 32 \\
40 & 36 \\
59 & 40 \\
36 & 33 \\
\hline
\end{tabular}


Table (4): Statistical analysis of group (B).

\begin{tabular}{lcc}
\hline Statistical data & Pre-operative & Post-operative \\
\hline Mean & 42.33 & 32.00 \\
SD & 13.71 & 11.08 \\
SEM & 3.96 & 3.20 \\
Number & 12 & 12 \\
\hline
\end{tabular}

\section{DISCUSSION}

Although the extensor mechanism of the hand seemingly simple in its anatomy and function, it is actually a complex set of interlinked muscles, tendons, and ligaments. The extensor apparatus is a linkage system created by ulnar and median nerves innervated intrinsic system and radial nerve innervated extrinsic system. The anatomy and function of the extensor mechanism of the hand are more intricate and complex than those of the flexor system [9]. Pre-operatively, ensuring patient education and participation in postoperative therapy is very important, bad outcomes are likely for patients who cannot tolerate post-operative physiotherapy. The hand surgeon must remember that non-operative treatment is of paramount importance [10].

The superficialis tenodesis technique uses the ulnar slip of the FDS is divided proximal to the decussation and left attached at its insertion and its proximal end is passed through a loop in the distal end of the $A_{2}$ pulley and sutured on itself to create a strong check to full extension of the PIP joint. If the $\mathrm{A}_{2}$ pulley is of poor quality, the slip can be attached to the bone of the proximal phalanx. This method does not rebalance the extensor mechanism at the distal joint [2]. If the patient desires both DIP and PIP correction, an oblique retinacular ligament reconstruction or lateral band tenodesis may be performed instead of tenodesis of the FDS [11].

Zancolli in 1979 advocated lateral band translocation. The lateral band on either radial or ulnar side of the finger is raised. The proximal part of the tendon is not divided and the tendon is raised as a bucket handle. The tendon is then translocated to the volar side and maintained in this position by suturing to the volar plate or the FDS or to $A_{2}$ pulley. This technique gives good and predictable results [12].

In this study on 20 fingers of Nalebuff type 1 (mobile joints not dependant on metacarpophalangyeal joint) [13], we reconstructed swan neck deformity by lateral band mobilization, the goal was to assess the use of a lateral band reconstruction on DIP extension lag and also PIP hyperextension. The results were very promising for short term follow up as the extension lag improved from mean preoperative 22.20 degree to 15.70 degree postoperative, with significant $p$-value $(0.0015)$ for 6 months follow up. Our results are confirming those reported by Tonkin in (1992) [11] despite a mean follow-up of 8 years, is much longer than our follow-up period, with Tonkin no patient experienced recurrence of the deformity or required reoperation and few fingers developed a slight slackening of the tenodesis with a decrease extension lag but no recurrence of the swan neck deformity. This procedure provides effective and sustained correction of swan neck deformity, with no functional impairment. The minimally invasive nature of the procedure, with no requirement for a tendon graft, expedites the rehabilitation process. The procedure is rapid and simple to be performed on several fingers during the same operation without complicating the postoperative rehabilitation program [13].

The long-term result of lateral band translocation is disappointing in Bruin (2010) [4] series. The success rate had decreased from $84 \%$ to $60 \%$. But we think different patient category of Bruin (cerebral palsy) has great impact on his results due to hand muscle atrophy. It should not be advocated as a procedure with long-lasting success in patients with cerebral palsy. The results have been generally favorable in the short term, but recurrent finger deformities with longer follow-up have been reported [1]. Progressive pannus formation in rheumatoid arthritis will lead to attenuation of soft tissues and joint deformity with a potential for relapsing instability [14]. We prefer a dual-slip lateral band transfer procedure as an option for treating chronic especially rheumatoid arthritis patients, post-traumatic hypextension instability of the PIP joint when the articulating surfaces and joint architecture are reasonably well preserved.

The concept of ORL reconstruction by linking the volar flexor sheath to the lateral aspect of the terminal tendon will improve the stability of both the DIP and PIP joints. Littler in [3] first described the use of an intact lateral band for ORL reconstruction, the band is released proximally then rerouted to volar side of the PIP joint and secured to the flexor tendon sheath. Thompson [7] used a free tendon graft to reconstruct the ORL. There have been no studies comparing these two ORL reconstruction techniques, and the advantage of one method over the other has not been adequately explored. Additionally, the issue of whether one 
method of bone fixation is superior to another has not been explored also.

Complications According to Nalebuff and Millender [6] include recurrence of the deformity caused by attenuation, excessive proximal interphalangeal joint flexion deformity. In this study on 12 fingers of Nalebuff type (1) [13] we reconstruct swan neck deformity by ORL reconstruction using tendon graft, the goal was to assess the reconstruction on DIP extension lag and also PIP hyperextension. The results were not promising with short term follow-up as the extension lag improved from mean preoperative 42.33 degree to 32.50 degree postoperative, with non-significant $p$-value .0578 for 6 months follow-up. The results are confirming those reported by Oh JY et al., [1] the overall improvement of approximately $10^{\circ}$ can be challenged in the interest of the operation. The ORL reconstructive technique did not show statistically significant differences in terms of functional outcome of DIP extension.

\section{Conclusions:}

Based on the findings of the present study, the following conclusions were made: There was significant difference between preoperative and early postoperative results (6 months) using lateral band mobilization especially with non-rheumatoid patients (burn and trauma), but the short and long term result of lateral band translocation in rheumatoid patients is disappointing in this study, as about $45 \%$ of our rheumatoid patient develop recurrent laggings on DIP joints and hyperextension of PIP joints with longer follow-up periods. We advocate separate studies of lateral band translocation in trauma patients and rheumatoid patients. As regard as ORL reconstruction with tendon graft, it did not show statistically significant results, the overall improvement is considered a challenge in the interest of the operation. We support continued trials for this technique in non-rheumatoid patients and with new modalities as bone fixation.

\section{REFERENCES}

1- Jae Yun Oh, Jin Soo Kim, Dong Chul Lee, Jae Won Yang, et al.: Comparative Study of Spiral Oblique Retinacular Ligament Reconstruction Techniques Using Either a Lateral Band or a Tendon Graft. Arachives of Plastic Surgery, 40: 6, 2013.

2- Knight S.L.: Assessment and management of swan neck deformity. International Congress Series, 1295: 154, 2006.

3- Littler J.W.: Restoration of the oblique retinacular ligament for correcting hyperextension deformity of the proximal interphalangeal joint, La Main Rheumatismale, L'Expansion Scientifique Francaise, Paris, 39, 1966.

4- De Bruin M., van Viet D.C., Smeulders M.J., et al.: Longterm results of lateral band translocation for the correction of swan neck deformity in cerebral palsy. J. Pediatr. Orthop., 30: 67, 2010.

5- Inoue G. and Tamura Y.: Dislocation of the extensor tendons over the metacarpophalangeal joints. J. Hand Surg. Am., 21 (3): 464, 1996.

6- Nalebuff E.A. and Millender L.H.: Surgical treatment of the swan-neck deformity in rheumatoid arthritis. Orthop. Clin. North Am., 6: 733, 1975.

7- Thompson J.S., Littler J.W. and Upton J.: The spiral oblique retinacular ligament (SORL), J. Hand Surg., 3: $482,1978$.

8- Rockwell W.B., Butler N.P. and Byrne A.B.: Extensor Tendon: Anatomy, Injury, and Reconstruction Salt Lake City, Utah, 106 (7): 1592, 2000.

9- Inoue G. and Tamura Y.: Dislocation of the extensor tendons over the metacarpophalangeal joints. J. Hand Surg. Am., 21 (3): 464, 1996.

10- Wei D.H. and Terrono An. L.: Superficialis Sling (Flexor Digitorum Superficialis Tenodesis) for Swan Neck Reconstruction. J. Hand Surg. Am., 40: 2068, 2015.

11- Tonkin M.A., Hughes J. and Smith K.L.: Lateral band translocation for swan neck deformity, J. Hand Surg., 17A: 260, 1992.

12- Charruaua B., Laulana J. and Saint-Castb Y.: Lateral band translocation for swan-neck deformity: Outcomes of 41 digits after a mean follow-up of eight years. Orthopaedics \& Traumatology: Surgery \& Research, 102: 221, 2016.

13- Nalebuff E.A.: The rheumatoid swan-neck deformity. Hand Clin., 5: 203, 1989. 